Virginia Commonwealth University VCU Scholars Compass

2005

\title{
Electroreflectance spectroscopy in self-assembled quantum dots: lens symmetry
}

\author{
A. H. Rodríguez \\ Universidad de La Habana, Universidad Autónoma de Puebla \\ C. Trallero-Giner \\ Universidad de La Habana \\ Martín Muñoz \\ Virginia Commonwealth University \\ María C. Tamargo \\ City College of the City University of New York
}

Follow this and additional works at: http://scholarscompass.vcu.edu/phys_pubs

Part of the Physics Commons

Rodríguez, A. H., Trallero-Giner, C., Muñoz, M., et al. Electroreflectance spectroscopy in self-assembled quantum dots: lens symmetry. Physical Review B, 72, 045304 (2005). Copyright (C) 2005 American Physical Society.

\section{Downloaded from}

http://scholarscompass.vcu.edu/phys_pubs/89

This Article is brought to you for free and open access by the Dept. of Physics at VCU Scholars Compass. It has been accepted for inclusion in Physics Publications by an authorized administrator of VCU Scholars Compass. For more information, please contact libcompass@vcu.edu. 


\title{
Electroreflectance spectroscopy in self-assembled quantum dots: lens symmetry
}

\author{
A. H. Rodríguez, ${ }^{1,2}$ C. Trallero-Giner, ${ }^{1}$ Martín Muñoz, ${ }^{3}$ and María C. Tamargo ${ }^{4}$ \\ ${ }^{1}$ Facultad de Física, Universidad de La Habana, 10400 C. Habana, Cuba \\ ${ }^{2}$ Instituto de Física, Universidad Autónoma de Puebla, Apdo. Postal J-48, Puebla, Pue. 72570, México \\ ${ }^{3}$ Physics Department, Virginia Commonwealth University, 1020 West Main Street Richmond, Virginia 23284, USA \\ ${ }^{4}$ Chemistry Department, City College of the City University of New York, Convent Avenue at 138th Street,
}

New York, New York 10031, USA

(Received 14 January 2005; published 5 July 2005)

\begin{abstract}
Modulated electroreflectance spectroscopy $\Delta R / R$ of semiconductor self-assembled quantum dots is investigated. The structure is modeled as dots with lens shape geometry and circular cross section. A microscopic description of the electroreflectance spectrum and optical response in terms of an external electric field (F) and lens geometry have been considered. The field and lens symmetry dependence of all experimental parameters involved in the $\Delta R / R$ spectrum have been considered. Using the effective mass formalism the energies and the electronic states as a function of $\mathbf{F}$ and dot parameters are calculated. Also, in the framework of the strongly confined regime general expressions for the excitonic binding energies are reported. Optical selection rules are derived in the cases of the light wave vector perpendicular and parallel to $\mathbf{F}$. Detailed calculation of the Seraphin coefficients and electroreflectance spectrum are performed for the InAs and CdSe nanostructures. Calculations show good agreement with measurements recently performed on $\mathrm{CdSe} / \mathrm{ZnSe}$ when statistical distribution on size and shape are considered, explaining the main observed characteristic in the electroreflectance spectra.
\end{abstract}

DOI: 10.1103/PhysRevB.72.045304

PACS number(s): 73.21.La, 78.67.Hc, 78.66.Hf, 73.22.-f

\section{INTRODUCTION}

Modulation spectroscopy is a technique based on the changes of the reflectivity of a sample when a periodic perturbation is applied. Due to its nature, this technique provides derivativelike spectra related to the optical transitions in the structure under consideration. Since its early stages in the $1960 \mathrm{~s}^{1-5}$ this technique has been providing valuable information about the properties of bulk/thin film semiconductors, reduced dimensional systems such as quantum wells and superlattices, ${ }^{6,7}$ and semiconductor device structures. ${ }^{8,9}$ In spite of the versatility and success of modulation spectroscopy few works have been done using this technique for the analysis of quantum wires ${ }^{10}$ and quantum dot (QD) structures. ${ }^{11,12}$ The optical properties of selfassembled QDs (SAQD) have been widely studied using photoluminescence, ${ }^{13,14}$ photoluminescence excitation spectroscopy, ${ }^{13,14}$ and time resolved photoluminescence. ${ }^{15}$ However, the information obtained is restricted to lower energy states and does not allow to study the shape of the QD potential or the coupling effects in stacked structures. Even though modulation spectroscopy allows to perform studies of lower and higher energy transitions in QD structures very little work has been performed on this subject. ${ }^{11,12}$

Reference 11 reported contactless electroreflectance (CER), which is a modulated technique that measures the changes in the optical reflectance of the material with respect to a modulating electric field, at room temperature in $\mathrm{CdSe}$ QDs with ZnSe barriers. The studied structure consists of a GaAs buffer layer followed by GaAs/AlAs short-period superlattice and CdSe QDs with ZnSe barriers (see inset in Fig. 1). The corresponding spectrum shows a profile related to the buffer layer, superlattices, QD region, wetting layer, and barriers. In the spectral region $\hbar \omega<2.2 \mathrm{eV}$ the typical Franz
Keldysh oscillations are present, which are fit using Lorentzian broadened electro-optical functions. ${ }^{6}$ Also, the structures originating above $2.6 \mathrm{eV}$ were fit using the first derivative of a Gaussian line shape. ${ }^{16}$ As shown in Fig. 1 the electroreflectance spectrum coming from the confined QDs region presents a broad structure which cannot be fit using

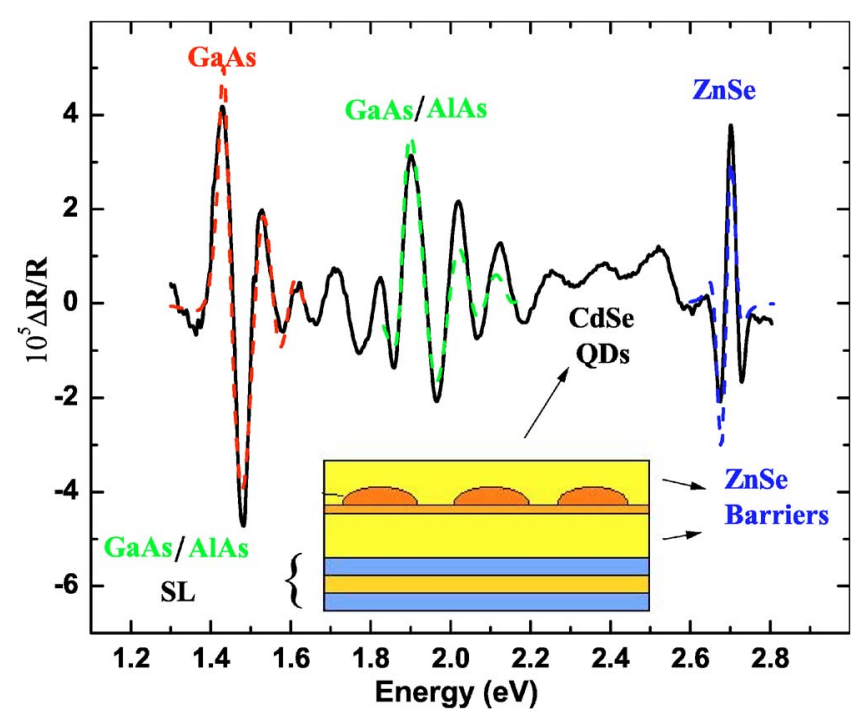

FIG. 1. (Color online) Contactless electroreflectance spectrum $\Delta R(\hbar \omega, F) / R(\hbar \omega, 0)$ for a CdSe quantum dot structure. Solid line corresponds to the spectral measurement. Dashed lines in the spectral region $\hbar \omega<2.2 \mathrm{eV}$ represent fit using standard electro-optical functions, while for the region above $2.6 \mathrm{eV}$ a fit using the first derivative of a Gaussian lineshape. The QDs spectral region addressed in this paper is $2.2<\hbar \omega<2.5$. The inset shows a typical $\mathrm{CdSe} / \mathrm{ZnSe}$ sample studied. 
standard electro-optical functions. ${ }^{1}$ It is clear that a reliable theoretical model for any modulation spectroscopy technique applied to quantum dots should include the primordial geometric factor. The modulation spectroscopy reflects doubtless the inherent quantum dot geometries.

In this paper we present a modulation spectroscopy study of quantum lens (QL) structures, based on CER. By full incorporation of the elements entering these experiments, namely, the effects of the geometry and electric field on excitonic states confined in the dot, the oscillator strengths, exciton energies, and Seraphin coefficients involved in the dielectric function, we provide the basis for quantitative analysis of CER in SAQD with lens symmetry. We study the effects of lens parameters on the electroreflectance spectrum $\Delta R / R$ and the optical response. We found that the lens symmetry has strong and clear signatures in the modulation spectroscopy, photoluminescence, and photoluminescence excitation spectroscopy. Moreover, we show that a detailed analysis of the optical response could provide information on the lens geometry and effective mass of the carriers, since they affect strongly the general features and overall peak distribution and amplitudes of the $\Delta R / R$ profiles. Since the modulation spectroscopy data had played a prevalent role in the study of III-V and II-VI semiconductors compounds, and because of its intrinsic interest, we present a detailed analysis of a model for a SAQD, which captures the essential physics of the problem. In fact, the lens symmetry is likely to be a good model of SAQDs, where the characteristic dimensions, height/diameter $<1$ are typical in these systems and our model should provide a good description.

The remainder of the paper is organized as follows. Section II deals with the general trends of the theory for $\Delta R / R$ applied to the case of a quantum lens, discussing the nature of the excitonic states in these structures and taking into consideration the external electric field effects. Section III presents theoretical calculations for the InAs/GaAs system, as well as the $\mathrm{CdSe} / \mathrm{ZnSe}$ system. Section IV contains a fit to the $\Delta R / R$ data in $\mathrm{CdSe} / \mathrm{ZnSe} \mathrm{SAQD}$ structures. Finally, Sec. $\mathrm{V}$ is devoted to the conclusions. In the appendixes, we present the behavior of the Seraphin coefficients for InAs/GaAs and $\mathrm{CdSe} / \mathrm{ZnSe}$ quantum lenses and some technical details of the calculations.

\section{BASIC RELATIONS}

A precise knowledged of the interband transition energies in a semiconductor can be traced by measuring the electroreflectance spectra. ${ }^{1}$ This spectroscopy technique is based on the modulation of an ac external electric field which modifies the shape of the dielectric function $\varepsilon(\hbar \omega) .{ }^{4}$ For normal incidence of the light the modulated electroreflectance $\Delta R / R$ is related to the real $\varepsilon_{1}$ and imaginary $\varepsilon_{2}$ parts of the dielectric function by

$$
\frac{\Delta R(\hbar \omega, F)}{R(\hbar \omega, 0)}=\alpha(\hbar \omega) \Delta \varepsilon_{1}(\hbar \omega, F)+\beta(\hbar \omega) \Delta \varepsilon_{2}(\hbar \omega, F),
$$

where $\Delta \varepsilon_{i}=\varepsilon_{i}(\hbar \omega, F)-\varepsilon_{i}(\hbar \omega, 0)(i=1,2), F$ is the intensity of the electric field, $\hbar \omega$ is the photon energy, and $\alpha, \beta$ are the Seraphin coefficients (see Appendix A).
Using the standard semiclassical approach to describe the interaction between light and matter, the imaginary part of the dielectric function for direct allowed transitions takes the form

$$
\begin{aligned}
\varepsilon_{2}= & 16 \pi \frac{a_{B}^{3}}{V_{0}} \frac{R_{y}^{2}}{(\hbar \omega)^{2} m_{0}} \sum_{\alpha_{e}, \alpha_{h}}\left|\int \Psi_{\alpha_{e}, \alpha_{h}}(r, r) d^{3} r\right|^{2} \\
& \times\left|\hat{\mathbf{e}} \cdot \mathbf{p}_{c v}\right|^{2} \frac{\gamma_{\alpha_{e}, \alpha_{h}}}{\left(\hbar \omega-E_{\alpha_{e}, \alpha_{h}}\right)^{2}+\gamma_{\alpha_{e}, \alpha_{h}}^{2}},
\end{aligned}
$$

where $a_{B}$ is the Bohr radius, $R_{y}$ is the Rydberg constant, $V_{0}$ is the effective volume taking place in the process, $m_{0}$ is the free electron mass, $\hat{\mathbf{e}}$ is the polarization vector of the incident light, $\mathbf{p}_{c v}$ is the interband optical matrix element between conduction $c$ and valence $v$ bands, $\gamma_{\alpha_{e}, \alpha_{h}}$ is the broadening parameter of the Lorentzian function. In the above equation $\left|\int \Psi_{\alpha_{e}, \alpha_{h}}(r, r) d^{3} r\right|^{2}$ are the oscillator strengths for the allowed interband transitions to the states $\Psi_{\alpha_{e}, \alpha_{h}}$ with energies $E_{\alpha_{e}, \alpha_{h}}$.

The Kramers-Kronig relations provide the real part of dielectric function $\varepsilon_{1}$, i.e.,

$$
\begin{aligned}
\varepsilon_{1}= & 1+16 \pi \frac{a_{B}^{3}}{V_{0}} \frac{R_{y}^{2}}{(\hbar \omega)^{2} m_{0}} \\
& \times\left.\sum_{\alpha_{e}, \alpha_{h}}\left|\int \Psi_{\alpha_{e}, \alpha_{h}}(r, r) d^{3} r\right|\right|^{2}\left|\hat{\mathbf{e}} \cdot \mathbf{p}_{c v}\right|^{2} \mathcal{L}\left(\hbar \omega, E_{\alpha_{e}, \alpha_{h}}\right),
\end{aligned}
$$

where

$$
\begin{aligned}
\mathcal{L}\left(\hbar \omega, E_{\alpha_{e}, \alpha_{h}}\right)= & \frac{E_{\alpha_{e}, \alpha_{h}}-\hbar \omega}{\left(\hbar \omega-E_{\alpha_{e}, \alpha_{h}}\right)^{2}+\gamma_{\alpha_{e}, \alpha_{h}}^{2}} \\
& +\frac{E_{\alpha_{e}, \alpha_{h}}+\hbar \omega}{\left(\hbar \omega+E_{\alpha_{e}, \alpha_{h}}\right)^{2}+\gamma_{\alpha_{e}, \alpha_{h}}^{2}}-\frac{2 E_{\alpha_{e}, \alpha_{h}}}{E_{\alpha_{e}, \alpha_{h}}^{2}+\gamma_{\alpha_{e}, \alpha_{h}}^{2}} .
\end{aligned}
$$

Two independent optical configurations are possible by choosing properly the direction of the light wave vector $\kappa$ with respect to the applied electric field. (i) $\kappa\|\mathbf{F}\| \hat{\mathbf{z}}$ and the vector of polarization $\hat{\mathbf{e}} \perp \hat{\mathbf{z}}$ which is the typical configuration used in CER experiments. Here, the three valence bands, light hole (lh), split off (so), and heavy hole (hh) can couple to the incident light. (ii) $\kappa$ perpendicular to $\mathbf{F} \| \hat{\mathbf{z}}$ chosen along the quantum lens growth direction and the vector polarization $\hat{\mathbf{e}} \| \hat{\mathbf{z}}$. In this case the $\mathrm{lh}$ and so valence bands will contribute to the optical spectrum.

\section{A. Electronic structure}

We will consider a typical SAQD with lens symmetry that presents a circular cross section of radius $a$ and height $b$. Electron-hole pairs (EHPs) are confined in the SAQD domain under an electric field $F$ parallel to its $\mathrm{z}$ axial symmetry axis. The exciton wave functions are taken as solutions of 


$$
\begin{aligned}
& {\left[-\frac{\hbar^{2}}{2 m_{e}^{*}} \nabla_{e}^{2}-\frac{\hbar^{2}}{2 m_{h}^{*}} \nabla_{h}^{2}-e \mathbf{F}\left(\mathbf{r}_{e}-\mathbf{r}_{h}\right)-\frac{e^{2}}{\epsilon\left|\mathbf{r}_{e}-\mathbf{r}_{h}\right|}\right] \Psi_{\alpha_{e}, \alpha_{h}}\left(\mathbf{r}_{e}, \mathbf{r}_{h}\right)} \\
& \quad=\left(E-E_{g}\right) \Psi_{\alpha_{e}, \alpha_{h}}\left(\mathbf{r}_{e}, \mathbf{r}_{h}\right),
\end{aligned}
$$

where $E_{g}$ is the gap energy, $\epsilon$ is the dielectric constant, and $m_{i}^{*}(i=e, h)$ is the quasiparticle effective mass. In the strong spatial confinement (electron-hole Coulomb interaction can be considered as a perturbation) and according to the axial symmetry of the quantum lens, the electron-hole pair wave function $\Psi_{\alpha_{e}, \alpha_{h}}$ is given by a product of electronic wave functions $\Psi_{i}\left(\rho_{i}\right) \exp \left(i m_{i} \phi_{i}\right)$. Here, $m_{i}$ is the $z$ component of the orbital angular momentum and functions $\Psi_{i}$ satisfy a bidimensional Schrödinger equation for quantum dots with lens-shape geometry in an electric field. Closed solutions of one-particle wave functions $\Psi_{N, m}$ and energy levels $E_{N, m}(N$ enumerates, for a fixed value of $m$, the electronic levels by increasing value of the energy) as a function of the applied electric field and lens shape geometry have been published elsewhere. ${ }^{17,18}$ The excitonic correction, appearing in Eq. (5) has been considered in first-order perturbation theory. It is possible to identify two cases.

(i) $m_{e}=m_{h}=0$, where the EH states are not degenerate and the excitonic correction is directly given by

$$
\Delta E_{0}=-\frac{2 e^{2}}{\epsilon} \sum_{l=0}^{\infty} \frac{I_{l}\left(m_{e}=0, m_{h}=0 ; m^{\prime}=0\right)}{2 l+1} .
$$

(ii) If $m_{e}=m_{h} \neq 0$ fourth-fold degeneracy of the EHP levels has to be considered and a $4 \times 4$ matrix for the exciton eingenvalue is obtained. By symmetry it follows that the total $z$ component of the EHP angular momentum $M=m_{e}$ $+m_{h}$ is preserved under the electron-hole correlation. For example, if $m_{e}, m_{h}= \pm 1$ the states with $M= \pm 2$ are degenerate with an energy equal to

$$
\Delta E_{0}=-\frac{2 e^{2}}{\epsilon} \sum_{l=0}^{\infty} \frac{I_{l}\left(m_{e}= \pm 1, m_{h}= \pm 1 ; m^{\prime}=0\right)}{2 l+1} .
$$

While the states with $M=0$ are decupled with energies

$$
\begin{aligned}
& \Delta E_{+}=\Delta E_{0}+\frac{2 e^{2}}{\epsilon} \sum_{l=0}^{\infty} \frac{I_{l}\left(m_{e}=1, m_{h}=-1 ;\left|m^{\prime}\right|=2\right)}{2 l+1}, \\
& \Delta E_{-}=\Delta E_{0}-\frac{2 e^{2}}{\epsilon} \sum_{l=0}^{\infty} \frac{I_{l}\left(m_{e}=1, m_{h}=-1 ;\left|m^{\prime}\right|=2\right)}{2 l+1} .
\end{aligned}
$$

$I_{l}$ are dimensionless functions given in the Appendix B. The energetic order $\Delta E_{-}<\Delta E_{0}<\Delta E_{+}$is preserved for any value of the applied electric field or lens geometry (see Fig. 2). Notice that the same behavior and equations are obtained for any values of the quantum numbers $m_{e}, m_{h} \neq 0$.

In Fig. 2 the first calculated excitonic energies $\operatorname{Eex}\left(N_{e}, m_{e} ; N_{h}, m_{h}\right)-E_{g}$ for CdSe quantum lens as a function of the dimensionless electric field $F / F_{0}$ are plotted $\left[F_{0}\right.$ $\left.=E_{0} /(|e| a), E_{0}=\hbar^{2} /\left(2 m_{e}^{*} a^{2}\right)\right]$. For the calculations we have used the values given in Table I. Two types of quantum lens are considered representing the weak [Fig. 2(a), $b / a=0.91$ ] and the strong [Fig. 2(b), $b / a=0.51]$ lens confinement do-
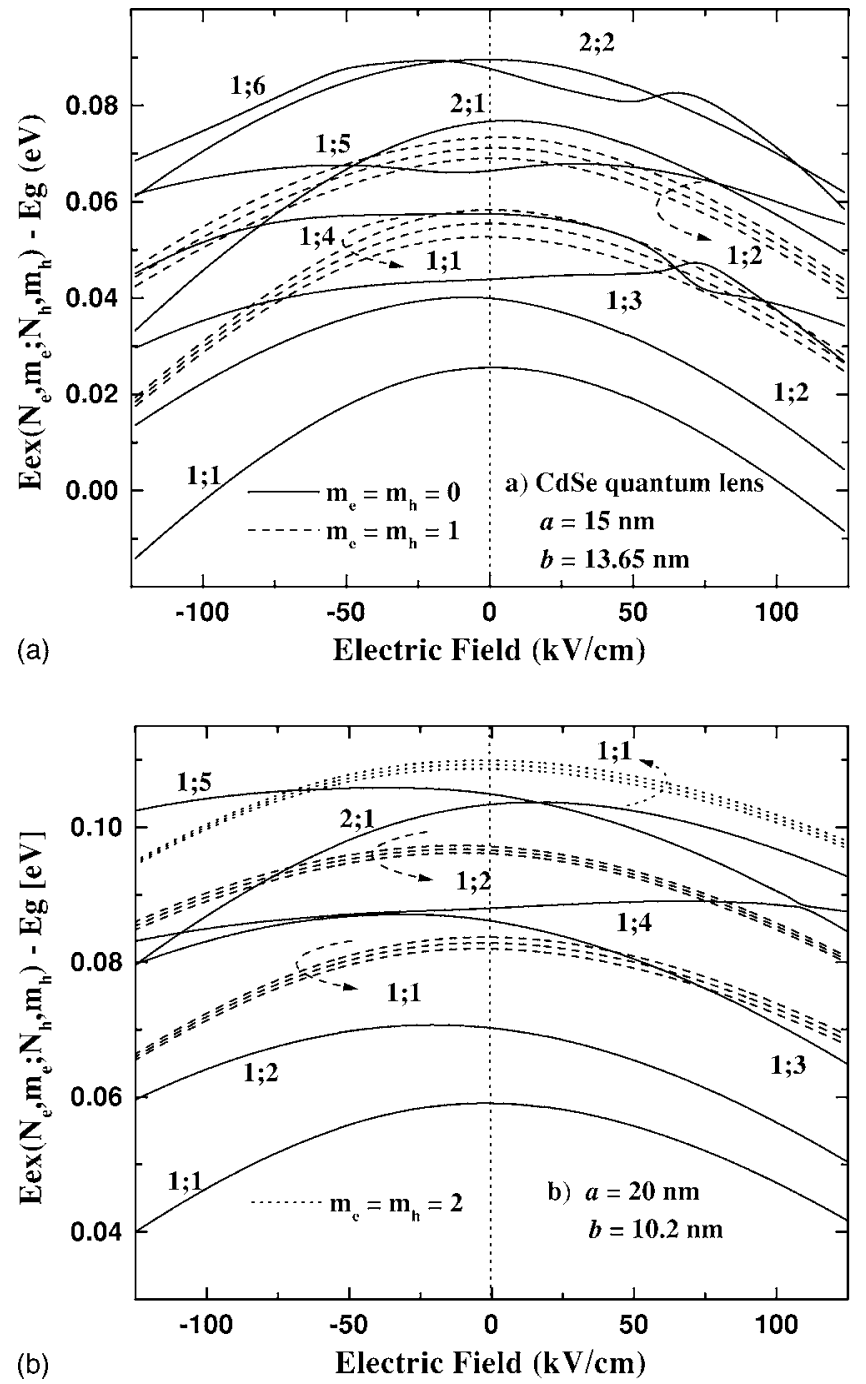

FIG. 2. Excitonic energy levels $\operatorname{Eex}\left(N_{e}, m_{e} ; N_{h}, m_{h}\right)-E_{g}$ for $\mathrm{CdSe}$ quantum lenses as a function of the electric field. Excitonic branches are labeled by $N_{e} ; N_{h}$ for the allowed optical transitions $\Delta m=m_{e}-m_{h}=0$. (a) Lens domain with $a=15 \mathrm{~nm}, b=13.65 \mathrm{~nm}$. (b) $a=20 \mathrm{~nm}, b=10.2 \mathrm{~nm}$.

mains, respectively. Excitonic states with $m_{e}=m_{h}=0,1$, and 2 are shown by solid, dashed, and dotted lines, respectively. In both calculations the excitonic correction represents a very small contribution to the total energy and the effect of the $F$ on $\Delta E$ is practically negligible. The splitting of the EHP levels, due to the electron-hole correlation, diminishes as the confinement increases. In the case of weak spatial confinement the electric field effect upon energetic levels is stronger as shown in Fig. 2(a) in comparison to Fig. 2(b). The interplay between $F$ and the ratio $b / a$ determines the peculiarities of the excitonic energy as a function of $F$ in particular on the excited states (for details see Ref. 18). Also, due to the lens geometry, the electronic energies present an asymmetric Stark shift with the external applied field.

\section{ELECTROREFLECTANCE}

In the following, we analyze the electroreflectance spectrum $\Delta R / R$ for the case of InAs/GaAs and $\mathrm{CdSe} / \mathrm{ZnSe}$ 
TABLE I. Parameters used in calculations.

\begin{tabular}{ccc}
\hline \hline Parameters & InAs & CdSe \\
\hline$E_{g}(\mathrm{eV})$ & $0.45^{\mathrm{a}}$ & $1.692^{\mathrm{b}}$ \\
$\epsilon$ & $14.6^{\mathrm{a}}$ & $9.3^{\mathrm{a}}$ \\
$m_{e}^{*} / m_{0}$ & $0.023^{\mathrm{a}}$ & $0.11^{\mathrm{b}}$ \\
$m_{\mathrm{hh}}^{*} / m_{0}$ & $0.34^{\mathrm{a}}$ & $0.44^{\mathrm{b}}$ \\
$m_{\mathrm{lh}}^{*} / m_{0}$ & $0.027^{\mathrm{a}}$ & - \\
$\Delta E_{c}(\%)$ & $40 \%^{\mathrm{a}}$ & $85 \%^{\mathrm{b}}$ \\
$\Delta E_{v}(\%)$ & $60 \%^{\mathrm{a}}$ & $15 \%^{\mathrm{b}}$ \\
$n_{\infty}$ & $3.517^{\mathrm{c}}$ & $2.5^{\mathrm{d}}$ \\
$P^{2} / m_{0}(\mathrm{eV})$ & $10.0^{\mathrm{c}}$ & $11.1^{\mathrm{c}}$ \\
$\gamma_{\mathrm{hh}}(\mathrm{meV})$ & 3 & 3 \\
$\gamma_{\mathrm{lh}}(\mathrm{meV})$ & 5 & \\
\hline
\end{tabular}

Ref. 21.

${ }^{\mathrm{b}}$ Ref. 11.

${ }^{\mathrm{c}}$ Ref. 22.

${ }^{\mathrm{d}}$ An average of the values reported in Ref. 22.

SAQDs. This measurement gives rise to sharp, differentiallike spectra in the region of the transitions. In the figures the main excitonic transitions are denoted by numbers $(1,2, \ldots)$, which correspond to a particular set of quantum numbers $\left(N_{e}, m_{e} ; N_{h}, m_{h}\right)$. Due to the axial symmetry, the interband selection rules correspond to excitonic branch with $\Delta m=m_{e}$ $-m_{h}=0$. The allowed transitions are resolved in the $\Delta R / R$ spectrum as different "effective gaps" and the peak positions are directly proportional to the lens geometry. In the case of $m_{e}=m_{h} \neq 0$ the EHP degeneracy is broken and additional structure appears in the electromodulation spectrum. We have only considered the incoming frequency in the range below the energy barrier, according to the material parameters listed in Table I. A full analysis of the electroreflectance response in each system provides complementary information to photoluminescence and photoluminescence excitation spectra to characterize the nanostructures involved and the quantum lens geometry.

\section{A. InAs/GaAs}

Figure 3 displays the electroreflectance spectra of InAs dots embedded in GaAs barriers for the cases of two independent optical configurations: (a) $\kappa \| \mathbf{F}$ and $\hat{\mathbf{e}} \perp \hat{\mathbf{z}}$ and (b) $\kappa \perp \mathbf{F}$ and $\hat{\mathbf{e}} \| \hat{\mathbf{z}}$. For the calculation the value of $E_{g}=1.51 \mathrm{eV}$ for GaAs has been used. Solid vertical arrows show the excitonic transitions for a lens geometry with $a=16.0 \mathrm{~nm}$ and $b=14.56 \mathrm{~nm}$ (solid lines), while dashed vertical arrows correspond to a QL with $a=20.5 \mathrm{~nm}, b=10.46 \mathrm{~nm}$. For the case of Fig. 3(a) we used the corresponding Seraphin coefficient $\alpha$ and $\beta$ displayed in Fig. 6(a), where the spatial confinement effect it can be noticed. Also for closely spaced peaks, the interference between different resonant levels increases and the $\Delta R / R$ signal is not simply the result of single contributions.

Due to the relative oscillator strength of the hh and $\mathrm{lh}$ valence bands, the electroreflectance features appear as relatively large resonant peaks in the case of $\kappa \| \mathbf{F}[$ Fig. 3(a)] in

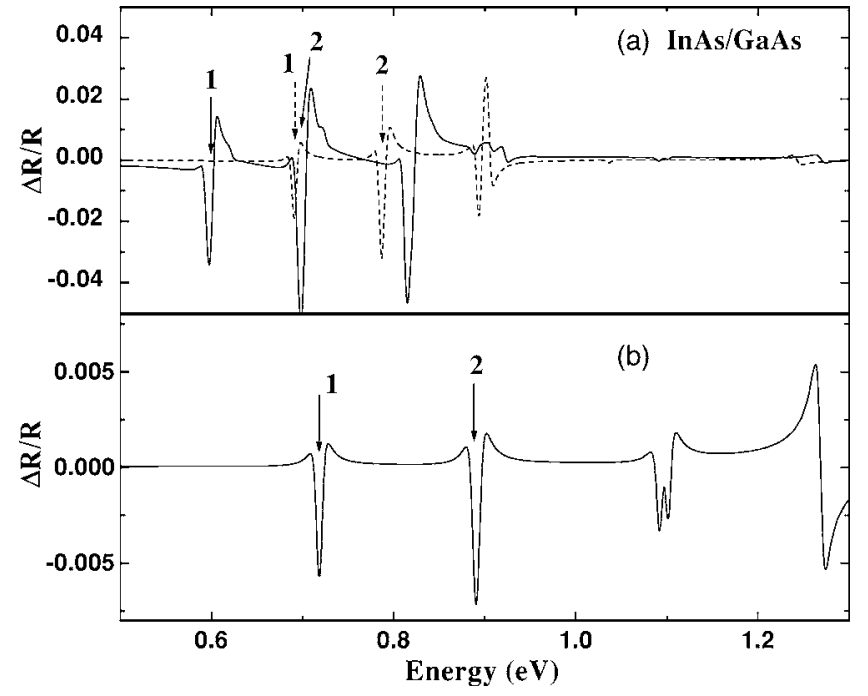

FIG. 3. Electroreflectance spectrum for InAs quantum lenses. Solid lines correspond to the lens domain $a=16 \mathrm{~nm}, b=14.56 \mathrm{~nm}$ and dashed lines to $a=20.5 \mathrm{~nm}, b=10.46 \mathrm{~nm}$. Optical configurations (a) $\kappa \perp \mathbf{F} \| \hat{\mathbf{z}}$ and $\hat{\mathbf{e}} \| \hat{\mathbf{z}}$. (b) $\kappa\|\mathbf{F}\| \hat{\mathbf{z}}$ and $\hat{\mathbf{e}} \perp \hat{\mathbf{z}}$. In the calculation a value of $F=50 \mathrm{kV} / \mathrm{cm}$ is used. Allowed excitonic optical transitions are indicated by arrows

comparison to the spectrum for the optical configuration $\kappa \perp \mathbf{F}, \hat{\mathbf{e}} \| \hat{\mathbf{z}}$ [Fig. 3(b)]. Labels 1 and 2 for all graphs correspond to the transitions between $N_{h}=1, m_{h}=0 \longrightarrow, N_{e}=1, m_{e}$ $=0$ and $N_{h}=1, m_{h}=1 \rightarrow, N_{e}=1, m_{e}=1$, respectively. Notice in particular that in Fig. 3(a) the transitions involving the hh exciton are substantially stronger and the light hole oscillator strength is about 10 times smaller than that corresponding to the heavy hole. In the configuration where $\kappa \perp \mathbf{F} \| \hat{\mathbf{z}}$ and $\hat{\mathbf{e}} \| \hat{\mathbf{z}}$ [Fig. 3(b)] the heavy hole excitonic branches are forbidden and labels 1 and 2 represent the light hole contributions.

\section{B. $\mathrm{CdSe} / \mathrm{ZnSe}$}

To illustrate the role of the II-VI materials that compose a QL, Fig. 4 shows the electroreflectance spectrum as a function of the photon energy for CdSe dots with $\mathrm{ZnSe}$ barriers. A value of $E_{g}=2.7 \mathrm{eV}$ for $\mathrm{ZnSe}$ is used for the numerical evaluation. The obtained spectra correspond to the cases of Fig. 2, solid line for a QL with $a=15.0 \mathrm{~nm} b=13.65 \mathrm{~nm}$, while dashed lines to the geometry with $a=20.0 \mathrm{~nm}, b$ $=10.20 \mathrm{~nm}$. To calculate $\Delta R / R$ we used the $\alpha$ and $\beta$ parameters shown in Fig. 6(b). The stronger oscillation strength is due to the excitonic branch $N_{e}=N_{h}$ and $m_{e}=m_{h}$, the rest of the allowed transitions are too weak to be resolved in ER spectrum. According to this, the exciton dispersion relations calculated in Fig. 2 closely follow the calculated $\Delta R / R$ structure. In general the spectra show the same general trend with respect to the InAs case. Nevertheless, two main differences are present: (i) Due to the heavy hole mass and for a given geometry the electroreflectance spectrum of CdSe has more structure that in the InAs QL. (ii) The exciton degeneracy is broken for $m_{e}=m_{h} \neq 0$ (displayed in Fig. 4 as a circle for the case of weak confined $b / a=0.91)$. Excitonic binding energies $\Delta E$, for II-VI semiconductors are larger than the III-V 


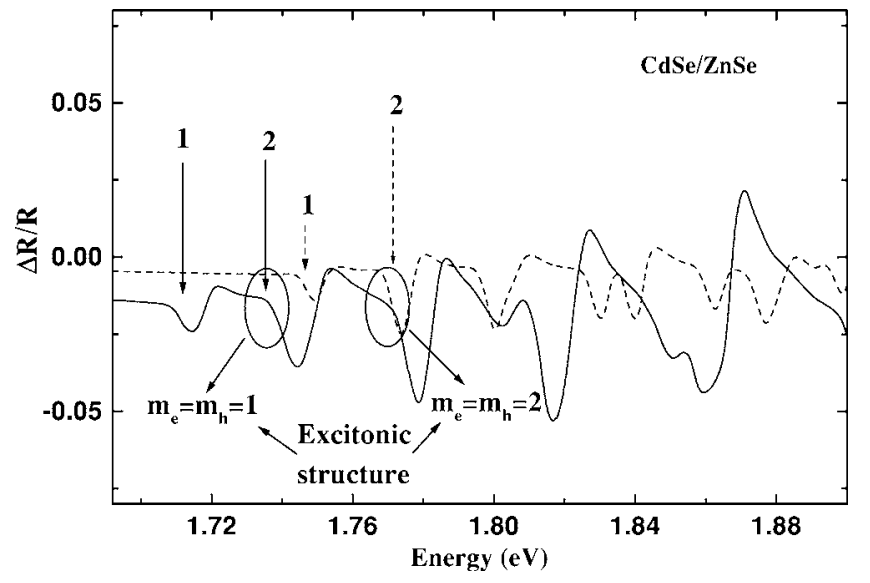

FIG. 4. Electroreflectance spectrum for CdSe quantum lenses for the optical configuration $\kappa\|\mathbf{F}\| \hat{\mathbf{z}}$ and $\hat{\mathbf{e}} \perp \hat{\mathbf{z}}$. Solid lines correspond to the lens domain $a=16 \mathrm{~nm}, b=14.56 \mathrm{~nm}$ and dashed lines to $a=20 \mathrm{~nm}, b=10.2 \mathrm{~nm}$. $F=50 \mathrm{kV} / \mathrm{cm}$ is used and the allowed excitonic optical transitions are indicated by arrows.

ones and consequently, the exciton degeneracy can be easily resolved by a spectroscopy technique.

\section{APPLICATION TO CADMIUM SELENIDE/ZINC SELENIDE QUANTUM LENS}

Figure 1 shows the experimental CER spectrum of $\mathrm{CdSe} / \mathrm{ZnSe} \mathrm{SAQD}$ at room temperature. ${ }^{11}$ We have performed calculations of the $\Delta R / R$ within the framework of the model developed in this paper, in order to compare its ability to reproduce the experimental data. A typical QD structure is shown in the inset of Fig. 1. Details about the growth conditions of the $\mathrm{CdSe} / \mathrm{ZnSe} \mathrm{QD}$ samples are provided elsewhere. ${ }^{11}$ The CER spectra of these structures have been obtained using a condenserlike system ${ }^{16}$ consisting of a front wire grid electrode with a second metal electrode separated from the first electrode by insulating spacers, which are approximately $0.1 \mathrm{~mm}$ larger than the sample dimension. The sample was placed between these two capacitor plates and the electromodulation was achieved by applying an ac voltage of $1.2 \mathrm{kV}, 200 \mathrm{~Hz}$ across the electrodes. In Fig. 1 we can identified the differential-like spectra originating from the QDs in the region $2.2<\hbar \omega<2.5 \mathrm{eV}$. In Fig. 5 electroreflectance data for the CdSe QD are displayed as solid circles. To fit the transitions originating from the QDs with QL geometry we took $a_{0}=11.98 \mathrm{~nm}$ and $b_{0} / a_{0}=0.24$. The solid line corresponds to the evaluation of Eq. (1) for a single quantum lens in presence of an electric field equal to $F$ $=50 \mathrm{kV} / \mathrm{cm}$ and a constant exciton broadening parameter $\gamma_{\alpha_{e}, \alpha_{h}}=8 \mathrm{meV}$. The sharp differential-like structure matches very well with the measured QL allowed optical transitions. These peaks correspond to electron-heavy hole transitions $N_{e}=1, m_{e}=0 \rightarrow N_{h h}=1, m_{h h}=0$ and $N_{e}=1, m_{e}=1 \rightarrow N_{h h}=1$, $m_{h h}=1$.

Self-assembled quantum dots have a distribution on size and shape. For a given photon energy $\hbar \omega$ we have to take into account the contribution of all quantum lenses that fulfil the resonance conditions $\hbar \omega=E_{\alpha_{e}, \alpha_{h}}$ and evaluate the average

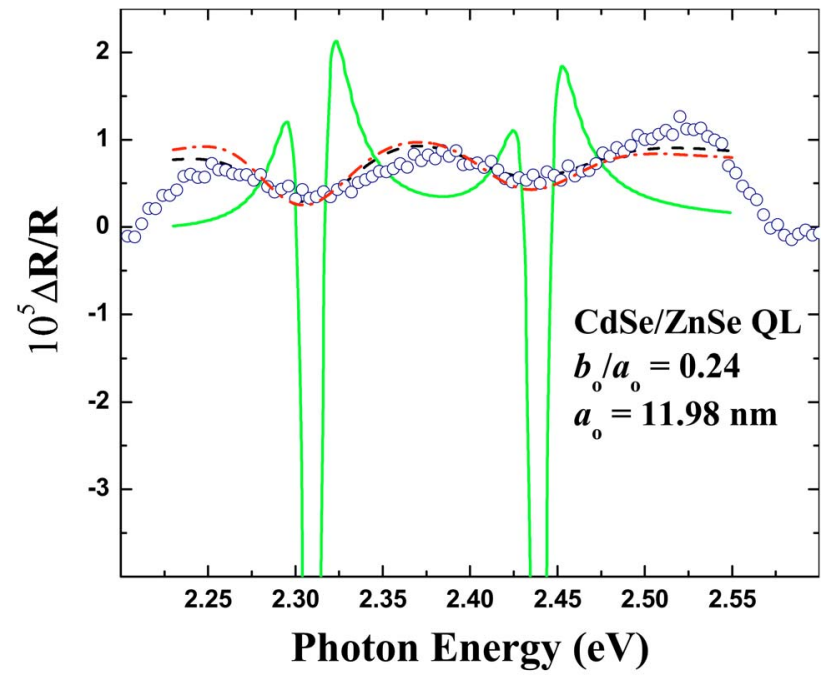

FIG. 5. (Color online) Contactless electroreflectance spectrum $\Delta R(\hbar \omega, F) / R(\hbar \omega, 0)$ for the $\mathrm{CdSe} / \mathrm{ZnSe}$ quantum dot structure shown in Fig. 1. Dots are the experimental data. The solid line represents the calculation for a QL with radius $a_{0}=11.98 \mathrm{~nm}$ and height $b_{0}=2.88 \mathrm{~nm}$, while dashed lines and dash dotted lines correspond to average size calculation by radius $a$ and ratio $b / a$ of $\overline{\Delta R(\hbar \omega, F) / R(\hbar \omega, 0)}$, respectively.

CER. In our calculation we have considered two independent statistical distribution. In the first one, we fixed the ratio $a_{0} / b_{0}$ and performed an average over the size $a$. In the second one, the radius $a_{0}$ was fixed and we average over the ratio $a_{0} / b_{0}$. The corresponding expressions for the average electroreflectance $\overline{(\Delta R / R)}$ are written as

$$
\overline{\left(\frac{\Delta R}{R}\right)}=\int F(a) \frac{\Delta R}{R} d a \text { or } \overline{\left(\frac{\Delta R}{R}\right)}=\int F\left(\frac{b}{a}\right) \frac{\Delta R}{R} d \frac{b}{a},
$$

where a Gaussian size distribution function $F(a)[F(b / a)]$ with mean value $a_{0}\left[a_{0} / b_{0}\right]$, and FWHM $\sigma_{a_{0}}\left[\sigma_{b_{0} / a_{0}}\right]$ is assumed. Figure 5 displays our theoretical calculations for the average $\overline{(\Delta R / R)}$ spectrum of an ensemble of CdSe quantum lens with average ratio $b_{0} / a_{0}=0.24, a_{0}=11.98 \mathrm{~nm}$. Dashed lines indicate the average spectrum by radius $a$ with $\sigma_{a_{0}}$ $=0.4 \mathrm{~nm}$, while dash dotted lines represent the distribution by ratio $b / a$ and $\sigma_{b_{0} / a_{0}}=3 \%$. In this particular case we observe that there are not significative difference in the $\overline{(\Delta R / R)}$ spectrum if we consider a distribution by the radius $a$ or by the ratio $b / a$. It can be seen that the observed measured broad spectrum is explained by size distribution of the QDs. Hence, the $\Delta R / R$ signal of Fig. 5 is the contributions of QDs in different resonance regimes, i.e., those excitonic transitions fulfilling the condition $\hbar \omega=E_{n_{e}, m_{e} ; n_{h}, m_{h}}(a, b)$. Resonances with higher exciton states occur for larger $a$ and $b$ values but are quenched by the size distribution function $F$ present in Eq. (10).

\section{CONCLUSIONS}

The present theoretical description can be used to evaluate the modulated electroreflectance spectra of III-V and II-VI 
SAQD with lens shape geometry. Optical responses and electroreflectance spectra as a function of the electric filed have been calculated for incoming photon energy above the fundamental effective gaps in QDs semiconductors. The Seraphin coefficients present a series of thresholds according to the excitonic $\left(N_{e}, m_{e} ; N_{h}, m_{h}\right)$ branch and the allowed optical transitions in the lens. The ER for InAs and CdSe dots show sharp differential-like spectra which identify the interband excitonic transitions of the QDs. The calculated $\Delta R / R$ dependence on $\hbar \omega$ reproduce quite well the experimental data available for $\mathrm{CdSe} / \mathrm{ZnSe}$ quantum dots. This fact indicates that the present theoretical model through out this paper contains the main ingredients of the electroreflectance spectroscopy in SAQD with lens geometry, and thus can be used, in combination with experimental data, to obtain information on the lens shape and other physical parameters related to the growth conditions of the sample. An important outcome of the work is that by fitting experimental data to this model we can estimate the size distribution of the QDs in the capped structures, which is a parameter not easily determined by other means.

\section{ACKNOWLEDGMENTS}

This work was partially supported by the National Science Foundation under Grant No. ECS0217646.

\section{APPENDIX A: SERAPHIN COEFFICIENTS}

These coefficients are related to the dielectric constant at zero electric field. Their spectral dependences are obtained by the expressions

$$
\begin{aligned}
& \alpha(\hbar \omega)=\frac{2 n}{n^{2}+k^{2}} \frac{n^{2}-3 k^{2}-1}{\left[(n+1)^{2}+k^{2}\right]\left[(n-1)^{2}+k^{2}\right]}, \\
& \beta(\hbar \omega)=\frac{2 k}{n^{2}+k^{2}} \frac{3 n^{2}-k^{2}-1}{\left[(n+1)^{2}+k^{2}\right]\left[(n-1)^{2}+k^{2}\right]} .
\end{aligned}
$$

The refractive index $n$ and the extinction coefficient $k$ are functions of $\varepsilon_{1}$ and $\varepsilon_{2}$ according to

$$
n=\sqrt{\frac{\sqrt{\varepsilon_{1}^{2}+\varepsilon_{2}^{2}+\varepsilon_{1}}}{2}}+n_{\infty}, \quad k=\sqrt{\frac{\sqrt{\varepsilon_{1}^{2}+\varepsilon_{2}^{2}}-\varepsilon_{1}}{2}},
$$

where $n_{\infty}$ is the refractive index at high frequency. Inserting Eqs. (2)-(4) at $F=0$ into Eqs. (A1)-(A3) we obtain the values of the Seraphin coefficients for the optical geometry $\kappa\|\mathbf{F}\| \hat{\mathbf{z}}$ and $\hat{\mathbf{e}} \| \hat{\mathbf{x}}$. Coefficients $\alpha$ and $\beta$ as a function of the photon energy for the InAs/GaAs and CdSe/ZnSe QLs are shown in Figs. 6(a) and 6(b), respectively. We have considered the same lens geometries indicated in Figs. 3 and 4. Weak and strong spatial confinement regimes are indicated by solid and dashed lines, respectively. Labels 1 and 2 represent the optical transitions between states $N_{h}=1, m_{h}=0$ to $N_{e}=1, m_{e}=0$ and $N_{h}=1, m_{h}=1$ to $N_{e}=1, m_{e}=1$. From the figures the strong influence of the lens geometry on the Seraphin coefficients and in consequence, on the electroreflectance, is clear.
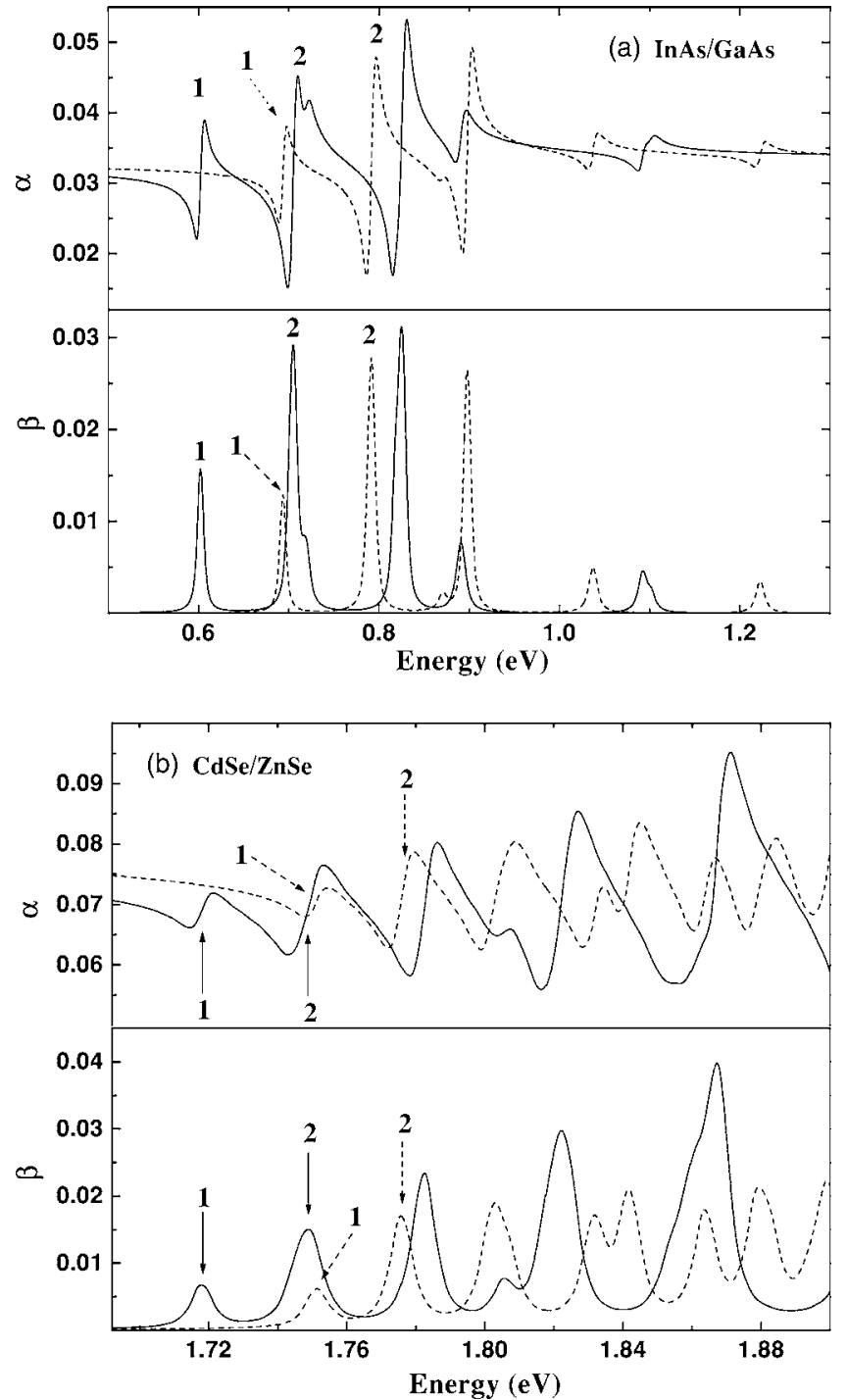

FIG. 6. Seraphin coefficients $\alpha$ and $\beta$ for a quantum lens in the optical configuration $\kappa\|\mathbf{F}\| \hat{\mathbf{z}}$ and $\hat{\mathbf{e}} \perp \hat{\mathbf{z}}$. The allowed excitonic optical transitions are indicated by arrows. (a) InAs/GaAs. (b) $\mathrm{CdSe} / \mathrm{ZnSe}$.

\section{APPENDIX B: EXCITON MATRIX ELEMENTS}

In Eqs. (6)-(9) the matrix element $I_{l}$ has an explicit expression (see Refs. 18 and 20):

$$
\begin{aligned}
I_{l}\left(m_{e}, m_{h} ; m^{\prime}\right)= & \sum_{i, j, i^{\prime}, j^{\prime}}^{\infty} C_{i}\left(N_{e}, m_{e}\right) C_{j}\left(N_{h}, m_{h}\right) C_{i^{\prime}}\left(N_{e}, m_{e}\right) \\
& \times C_{j^{\prime}}\left(N_{h}, m_{h}\right)\left\langle f_{i, m_{e}}^{(0)} f_{j, m_{h}}^{(0)}\right| \frac{r_{<}^{l}}{r_{>}^{l+1}} P_{l}^{\left|m^{\prime}\right|}\left(\cos \theta_{e}\right) \\
& \times P_{l}^{\left|m^{\prime}\right|}\left(\cos \theta_{h}\right)\left|f_{i^{\prime}, m_{e}}^{(0)} f_{j^{\prime}, m_{h}}^{(0)}\right\rangle,
\end{aligned}
$$

where coefficients $C_{i}(N, m)$ and functions $f_{i, m}^{(0)}$ are defined in Ref. 18. $I_{l}$ depends on the lens deformation $b / a$ and dimensionless electric field $F / F_{0}$. The excitonic correction integrals in Eq. (B1) were obtained by a Monte Carlo algorithm over the two-dimensional lens domain. 
${ }^{1}$ M. Cardona, Modulation Spectroscopy, Solid State Physics (Academic, New York 1969), Suppl. 11.

${ }^{2}$ M. Cardona, Festkorperprobleme X (Pergamon, Oxford, 1970), p. 125.

${ }^{3}$ Y. Hamakawa and T. Nishino, in Optical Properties of Solids: New Developments, edited by B. O. Seraphin (North-Holland, Amsterdam, 1976), p. 255.

${ }^{4}$ D. E. Aspnes in Handbook on Semiconductors, edited by M. Balkanski (North-Holland, Amsterdam, 1980), Vol. 2, p. 109.

${ }^{5}$ F. H. Pollak, Proc. Soc. Photo-Opt. Instrum. Eng. 276, 142 (1981).

${ }^{6}$ F. H. Pollak and H. Shen, Mater. Sci. Eng., R. 10, 275 (1993).

${ }^{7}$ O. J. Glembocki and B. V. Shanabrook, in Semiconductors and Semimetals, edited by R. K. Willardson and A. C. Beer (Academic, New York, 1992), Vol. 36, p. 221.

${ }^{8}$ F. H. Pollak, Mater. Sci. Eng., B 80, 178 (2001).

${ }^{9}$ F. H. Pollak, W. Krystek, M. Leibovitch, M. L. Gray, and W. S. Hobson, IEEE J. Sel. Top. Quantum Electron. 1, 1002 (1995).

${ }^{10}$ M. Geddo, S. Di Lernia, A. Stella, A. Bosacchi, S. Franchi, M. Gentili, and D. Peschiaroli, Solid State Commun. 100, 221 (1996).

${ }^{11}$ M. Muñoz, S. P. Guo, X. Zhou, M. C. Tamargo, Y. S. Huang, C. Trallero-Giner, and A. H. Rodríguez, Appl. Phys. Lett. 83, 4399 (2003).

${ }^{12}$ H. Qiang, F. H. Pollak, Y. S. Tang, P. D. Wang, and C. M. Sotomayor Torres, Appl. Phys. Lett. 64, 2830 (1994); F. H. Pollak, in Nano-Optoelectronics, edited by M. Grundmann (SpringerVerlag, Berlin 2002), p. 215.

${ }^{13}$ K. H. Schmidt, G. Medeiros-Ribeiro, M. Oestreich, and P. M. Petroff, Phys. Rev. B 54, 11346 (1996).

${ }^{14}$ R. Heitz, M. Grundmann, N. N. Ledentsov, L. Eckey, M. Veit, D. Bimberg, V. M. Ustinov, A. Yu. Egorov, A. E. Zhukov, P. S. Kop'ev, and Zh. I. Alferov, Appl. Phys. Lett. 68, 361 (1996).

${ }^{15}$ S. Raymond, S. Fafard, P. J. Poole, A. Wojs, P. Hawrylak, S. Charbonneau, D. Leonard, R. Leon, P. M. Petroff, and J. L. Merz, Phys. Rev. B 54, 11548 (1996).

${ }^{16}$ F. H. Pollak, in Group III Nitride Semiconductor Compounds, edited by B. Gil (Clarendon, Oxford, 1998), p. 158.

${ }^{17}$ Arezky H. Rodríguez, Carlos R. Handy, and C. Trallero-Giner, J. Phys.: Condens. Matter 15, 8465 (2003).

${ }^{18}$ Arezky H. Rodríguez and C. Trallero-Giner, J. Appl. Phys. 95, 6192 (2004).

${ }^{19}$ The volume of a quantum lens is given by $V=\pi a^{3}\left[b_{0} / a_{0}+(1 / 3)\right.$ $\left.\times\left(b_{0} / a_{0}\right)^{3}\right] / 2$ and for fixed ratio of $b_{0} / a_{0}$ the distribution in size $a$ is directly related to a distribution in volume.

${ }^{20}$ J. D. Jackson, Classical Electrodynamics (Wiley, New York, 1999).

${ }^{21}$ E. Menéndez-Proupin, C. Trallero-Giner, and S. E. Ulloa, Phys. Rev. B 60, 16747 (1999).

${ }^{22}$ Landolt-Börnstein Numerical Data and Functional Relationships in Science and Technology, edited by O. Madelung, New Series, Group III, Vol. 22 (Springer, Berlin, 1986). 